\title{
Osteoclastogenesis in Periodontitis: Signaling Pathway, Synthetic and Natural Inhibitors
}

\author{
Ketherin ${ }^{1}$, Ferry Sandra ${ }^{2,3}$ \\ ${ }^{1}$ Faculty of Dentistry, Trisakti University, Jakarta, Indonesia \\ ${ }^{2}$ Department of Biochemistry and Molecular Biology, Division of Oral Biology, Faculty of Dentistry, Trisakti University, Jakarta, \\ Indonesia \\ ${ }^{3}$ BioCORE Laboratory, Faculty of Dentistry, Trisakti University, Jakarta, Indonesia
}

\begin{abstract}
Osteoclast activities are responsible for the resorption of bone cells found in several bone diseases, one of which is periodontitis and arthritis. The upregulating signals of Receptor Activator of Nuclear Factor $\kappa B$ (RANK)-RANK Ligand and Tumor Necrosis Factor (TNF)- $\alpha$ are the major cause of the bone destruction. Studies and experiments have been performed to overcome this matter. Various medications are now available to treat bone-related diseases, targeting the specific aspect of the signaling. Synthetic drugs such as denosumab and bisphosphonates have complex pharmacological action and have been the leading choice in treatment. Evidence in studies proved that natural resources including herbal products have potential application to therapy for bone loss, with caffeic acid and Caffeic Acid Phenethyl Ester (CAPE) showing significant inhibitory results and Chinese herbs such as Herba epimedii (Yín Yáng Huò) and Fructus psoraleae (Bǔ Gǔ Zhī) proved to contain components that give similar effects to estrogen. The purpose of this review is to discuss the therapy value of available synthetic and natural therapeutic agents. Understanding the mechanisms of both agents will not only clarify their function as therapeutic agents, but can also be the key to the treatment of diseases caused by bone resorption by targeting specific aspects of osteoclastogenesis.
\end{abstract}

Keywords: osteoclastogenesis, TNF, RANKL, bone resorption, natural resource, signaling, treatment

\section{Introduction}

The bone loss progression seen in periodontitis is a result of excessive cytokine production, one of which is Receptor Activator of Nuclear Factor $\kappa B$ (RANK) Ligand (RANKL). RANK and RANKL signaling plays a prominent role in osteoclastogenesis, producing osteoclast cells. The binding of RANK-RANKL signaling induces Tumor Necrosis Factor (TNF) Receptor Associated Factor (TRAF) 6 and further leads to activation of its cascades such as

Date of submission: May 7, 2017

Accepted for publication: May 29, 2017

\section{Corresponding Author:}

Ferry Sandra

Department of Biochemistry and Molecular Biology, Division of Oral Biology

Faculty of Dentistry, Trisakti University, Jl. Kyai Tapa No.260

Jakarta, Indonesia

E-mail: ferrysandra@gmail.com
Extracellular Signal-Regulated Kinase (ERK), Janus N-terminal Kinase (JNK), Nuclear Factor $\kappa B(N F \kappa B)$, and p38 Mitogen-activated Protein Kinase (MAPK). ${ }^{1}$ The p38 MAPK has important roles in cell's homeostasis such as cell proliferation, differentiation, maturation, apoptosis, and cytokine production, which makes p38 MAPK one of the key factors in osteoimmunology. ${ }^{2}$ Therefore a therapeutic agent is needed to subdue this problem, and there is now evidence showing that not only synthetic medications but natural products can influence by inhibiting bone resorption

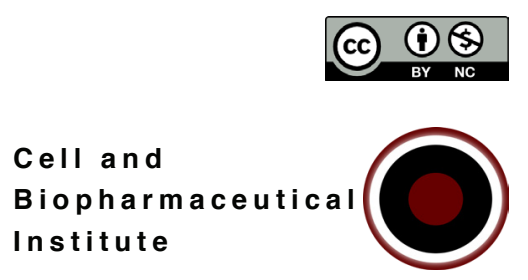


process. Vegetables including onion, garlic, and parsley were reported to inhibit bone resorption in ovariectomized rats, while herbs like thyme, rosemary, and sage, increase bone mineral density. ${ }^{3}$ Caffeic acid is a natural resource that has many uses as a therapeutic agent, one of which is to inhibit N-Nitrosation in vitro to inhibit the formation of mutagens and carcinogens. A study in 2011 showed that caffeic acid successfully suppresses osteoclastogenesis significantly by suppressing the activity NFkB. ${ }^{4}$ Another natural source is Stewartia koreana was reported to suppress osteoclast differentiation successfully. ${ }^{5}$ Drugs developed from synthetic substances including Bisphosphonates, Denosumab, Methotrexate (MTX) and Risedronate were also reported to have positive effects as a therapeutic agent of bone resorption related diseases. ${ }^{6,7}$ This review aims to introduce therapeutic options available for bone resorption diseases. Understanding the options and mechanisms will not only clarify their function as therapeutic agents but can also be the key to the treatment of diseases caused by bone resorption.

\section{Periodontitis}

Periodontitis defined by the American Academy of Periodontology as inflammation of the teeth supporting tissues that are usually characterized by progressive destructive changes that cause bone and periodontal ligament loss and an inflammatory response of the gingiva to the bone and ligaments adjacent. ${ }^{8}$ This disease is an opportunistic infection caused by plaque. Plaque is a mass that mostly composed of microorganisms that stick to the teeth, prosthesis, and the surface of the mouth. The roles of plaque containing these microorganisms have been analyzed many years and are now introduced to the concept called osteoimmunology, which means researchers are focusing their study on the signaling system between the immune system and bone cells.' In periodontal diseases, anaerobic bacteria and spirochetes grow in subgingival tissue then multiply into colonies and invade the host, generating irritation reactions and signs of inflammation. Extracts of Aggregatibacter actinomycetemcomitans has bacterial lipopolysaccharide (LPS) that induces a potent chemokine called interleukin (IL)-8. Induction of this chemokine will lead dendritic cells, neutrophils, macrophages, and lymphocytes to infiltrate the tissues and initiate the immune system. Stimulation of the bacteria then produces an inflammatory response in the form of excessive cytokine formation, which will initiate and exacerbate periodontal diseases. ${ }^{1}$

The initial stage of infection begins with the activation of a mechanical shield that acts as a non-specific immune defense system leading to initial inflammatory response. $\mathrm{B}$ and $\mathrm{T}$ lymphocytes soon are activated when the bacteria begins to invade the tissues. A study states that the researchers are considering the $\mathrm{T}$ lymphocytes as an important bone regulator not only in periodontitis but also in other cases. T lymphocytes activate macrophages, indirectly activating the expression of osteoclast precursors and RANKL. Current knowledge shows that RANK/RANKL/ Osteoprotegerin (OPG) signaling is involved in bone resorption process in patients suffering from periodontal disease. RANKL promotes bone resorption, while OPG inhibits bone resorption. Several studies were carried out to examine the levels of RANKL and OPG in patients with periodontitis. It was reported that patients with patients with periodontitis had lower levels of OPG. ${ }^{10}$ However, a study in ameloblastoma cell line showed that in a situation where OPG, TRAIL, and low level of RANKL present, OPG preferably binds to TRAIL, suppressing it's ability to induce apoptosis in ameloblastoma. TRAIL has the highest apoptotic ability compared to TNF- $\alpha$ and RANKL, making the existence of OPG should be considered. ${ }^{10}$ In addition to RANKL, TNF- $\alpha$ also plays an important role in inducing inflammation that exacerbates periodontitis. ${ }^{11}$ Based on the data above, it is clear that pro-inflammatory mediators are the main cause of the increased severity of periodontal diseases and many of these cytokines are obtained from activated p38 MAPK whose activities depend on phosphorylation of tyrosine and threonine. ${ }^{1}$

\section{Osteoclastogenesis}

Bone is a dynamic organ where it is constantly changing to reach its normal function called the bone remodeling process. Old bone cells will be degraded by the osteoclasts and replaced with new ones by osteoblasts. Therefore, the balance between bone formation and resorption process is vital to maintain bone's normal state. ${ }^{12}$ Osteoclast is a multinucleated cell whose function is to resorp bone, derived from hematopoietic stem cells, and is made of myeloid progenitor cells involving fused mononuclear cells. ${ }^{4,13}$ Osteoclast precursors undergo a series of differentiation stages resulting mature cells capable of destroying the bone. 
Hematopoietic stem cells are divided into two major groups, common myeloid progenitor (CMP) and the common lymphoid progenitor (CLP). Osteoclast comes from CMP, a bifurcation from monocyte/macrophage lineage while CLP induces lymphoid lineage cells such as T lymphocytes, B lymphocytes and other natural killer cells. ${ }^{15}$ A study showed that osteoblastic cells are also required for osteoclast differentiation. Osteoblastic cells produce Macrophague Colony Stimulating Factor (M-CSF), even though M-CSF alone is not sufficient to induce osteoclastogenesis. M-CSF enhances the proliferation and survival of osteoclast precursors and helps the survival of mature osteoclast cells. ${ }^{14}$

\section{RANK-RANKL Signaling}

The binding of RANK and RANKL is one of the major factors that affect osteoclastogenesis, proved in a study on mice. Osteopetrosis was seen in mice that had a shortage in RANK and RANKL induction. ${ }^{15}$ RANKL is a member of the TNF superfamily, which is a protein found on the surface of mesenchymal osteoblast lineage, binding to its receptor, RANK, which will activate the osteoclast phenotype. Production of RANKL can be increased by parathyroid hormones that act as a stimulant agent of the osteoclast. ${ }^{16}$ RANK does not have intrinsic enzyme activity in its intracellular domain; it converts the energy to recruit adapter molecules such as proteins TRAF family to overcome this. TRAF is a cytoplasmic adapter protein that binds to the intracellular domain of various receptor of TNF receptor (TNFR). ${ }^{17}$

Among all TRAFs, TRAF 6 is the most important adapter for RANK-RANKL to induce osteoclastogenesis. ${ }^{18}$ The relationship between TRAF6 and RANK will form a complex signaling, Transforming Growth Factor (TGF)$\beta$-activated kinase (TAK) 1 and TAK1-binding protein 2 which induce 3 MAPK signaling pathways such as ERK, p38 MAPK and JNK (Figure 1). The induction of TRAF6 in the RANK signaling is crucial in the phosphorylation process of RANK-induced NFKB. ${ }^{19}$ TRAF 6 will continue the signals to NFkB, ERK, p38 MAPK, JNK, Akt, c-Fos, c-Src and Microphthalmia-inducing Transcription Factor (MITF). ${ }^{20,21}$ RANKL induces Nuclear Factor of Activated $\mathrm{T}$ cells (NFATc) 1 complex by inducing the expression of the c-Fos family and supporting c-Jun signaling. ${ }^{22}$ NFATc1 regulates several osteoclast-specific genes, including the tartrate-resistant acid phosphatase, osteoclast-associated receptor and cathepsin $\mathrm{K}^{22}$ The important roles of NFATc1 in osteoclastogenesis have been studied with in vitro and in vivo tests carried out on mice. In in vitro studies, human embryonic stem cells that lack NFATc1 failed to differentiate into osteoclast, and in vivo observations, the

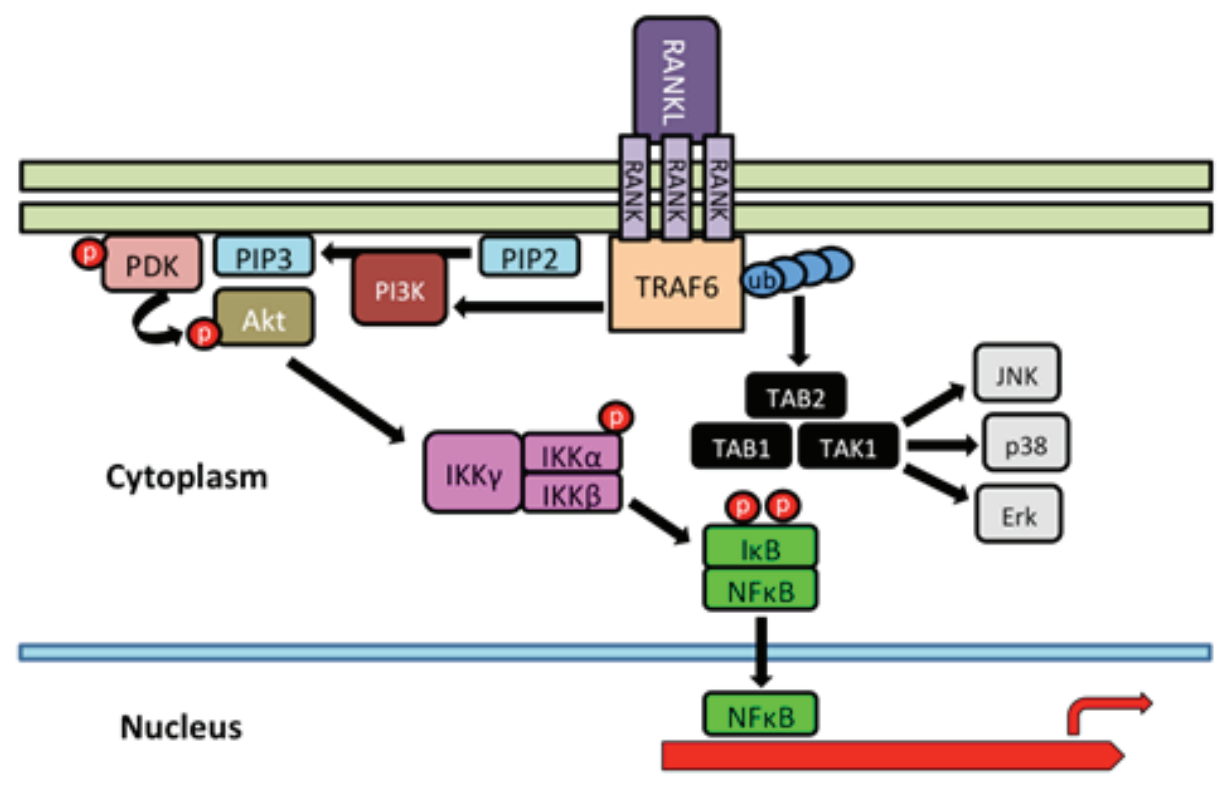

Figure 1. RANK-RANKL osteoclastogenesis signaling pathway. PIP2: phosphatidylinositol (4,5)-bisphosphate; PIP3: phosphatidylinositol $(3,4,5)$-trisphosphate; TAB: TAK1-binding protein; PDK: phosphoinositide-dependent protein kinase; I $\mathrm{B}$ : Inhibitor of $\kappa \mathrm{B}$; IKK: IкB kinase. 
absence of NFATc1 in young mice would result in osteopetrosis proving that NFATc1 plays an important role on osteoclastogenesis. ${ }^{23}$

RANKL also activates Phosphoinositides 3-kinase (PI3K)/Akt through TRAF6, PI3K/Akt has a close relationship with Src kinase activity. ${ }^{24}$ The absence of cellular Src kinase will inhibit phosphorylation of RANKL-induced Akt. Activated PI3K then creates phosphatidylinositol$(3,4,5)$-phosphate in the plasma membrane, which then recruits Akt. PI3K inhibitor LY294002 was reported to inhibit osteoclast formation. The data above shows that the signaling of PI3K is involved in the differentiation process of the osteoclast. ${ }^{25}$

\section{TNF Signaling}

While RANK-RANKL signaling is a major signaling in osteoclastogenesis, proinflammatory cytokines together with RANK and RANKL play an important role in osteoclast's differentiation and activation. ${ }^{26}$ TNF- $\alpha$ also induces osteoclast formation in vitro by activating the $\mathrm{NF \kappa B}$ and JNK. In fact, in vitro studies have hypothesized that TNF- $\alpha$ may induce osteoclastogenesis even when RANK-RANKL signaling is not present. TNF- $\alpha$ is formed by macrophages, monocytes and lymphocytes T. A study in mice showed an increased formation of osteoclast precursors in mice that had higher concentrations of TNF- $\alpha$. TNF- $\alpha$ is part of TNF superfamily and plays an important role in various cell activities such as cell proliferation, differentiation, maintenance and apoptosis. ${ }^{27} \mathrm{TNF}-\alpha$ induces paired Ig-like receptor-A (PIR-A), which is a co-stimulatory receptor for RANK to stimulate osteoclastogenesis. ${ }^{28}$

TNF- $\alpha$ also induces osteoclast precursor and marrow stromal cells that will express TNF receptor type 1 and produce proinflammatory cytokines, such as IL-1, RANKL and M-CSF. M-CSF then binds to c-fms, a tyrosine kinase receptor that induces osteoclast differentiation by activating ERK1/2 and PI3K/Akt giving direct impact on osteoclastogenesis. ${ }^{29}$ Great recognition has been given towards TNF- $\alpha$ as numerous studies about anti-TNF treatment showed promising results in reducing bone erosions and inflammation. ${ }^{30,31}$

\section{M-CSF}

Osteoblasts or the cells derived from new bones express 2 main cytokines contributing to osteoclast differentiation,
RANKL and M-CSF. Osteoclast precursors express M-CSF's receptor called c-Fms and RANK, which is RANKL's receptor. Their bindings play a major role in osteoclast differentiation. ${ }^{32} \mathrm{M}-\mathrm{CSF}$ is a molecule that plays a big role in precursor's survival and proliferation. A study confirmed that op/op mice that failed to express functioning M-CSF are osteopetrosis. Another study mentioned mice lacking csf1r or the gene coding for c-Fms, experience a decrease in tissue macrophages and severe osteopetrosis confirming the evidence above. ${ }^{33}$ The binding of M-CSF and c-fms has a complex downstream signals, however, to simplify it, $\mathrm{M}-\mathrm{CSF}$ induces signaling in primary macrophages such as PI3K, p42/44 ERK, and c-Cbl. These are key transducers of M-CSF signaling. ${ }^{34}$

\section{Available Bone Resorption Inhibitor}

The normal function of bone metabolism is deeply influenced by the balance of bone mineralization and resorption. However, the balance needed in maintaining bone's health is often compromised due to aging and bone related diseases. Thus, researchers have been studying prospecting materials to be developed as a potential therapy for bone related diseases.

\section{Synthetic Resources as Bone Resorption Inhibitor}

Resorption inhibitor drugs have been the most common therapy when it comes to dealing with bone related diseases. Drugs can influence osteoclast's activity in many ways with their own biochemical mechanisms, many of which are created to help reduce bone resorption thus helping the healing process of the bone. Treatments have been varied from hormones such as estrogens and calcitonin to Denosumab which is an anti-RANKL antibody. ${ }^{35}$

\section{Bisphosphonates}

Bisphosphonates are potent bone resorption inhibitors and have been used as primary and therapeutic agents for bone related diseases such as osteogenesis imperfecta, heritable skeletal disorderinchildren, postmenopausal, glucocorticoidinduced osteoporosis, and bone metastases in malignancy cases. Bisphosphonates inhibit bone's calcification and hydroxyapatite breakdown making it an effective bone resorption inhibitor. Newer generations of bisphosphonates such as alendronate, risedronate, ibandronate, pamidronate, 
and zoledronic acid have nitrogen in their chains by which promote osteoclast apoptosis more efficiently compared to non-nitrogen bisphosphonates. ${ }^{36}$ Further study showed that alendronate and pamidronate inhibited osteoclastogenesis but did not target RANKL and OPG expression. ${ }^{37}$ They inhibit farnesyl diphosphate synthase which is a cholesterol biosynthesis pathway enzyme, and by suppressing geranylgeranylation, osteoclast function is lost, and bone resorption progress is inhibited. ${ }^{38}$ However, prolonged consumption of bisphosphonates may increase the potential for diseases such as osteonecrosis of the jaw, atypical fractures, and esophageal cancer. ${ }^{39}$

\section{Trichostatin A}

Trichostatin A is one of histone deacetylases inhibitor, which is an enzyme involved in the transcriptional activity and the remodeling of chromatin's structure. A study was conducted to analyze the effects of TSA on RANKL induced osteoclast formation and results showed a significant decrease of c-Fos and NFATc1 which play a big role in early osteoclastogenesis. ${ }^{40}$ Further evidence showed TSA leads mature osteoclasts into apoptotic cell death thus making it a suitable candidate as a bone related therapeutic agent. ${ }^{41}$ Lastly, TSA was reported to successfully inhibit osteoclast formation in co-culture of bone marrow cells and osteoblasts without reducing RANKL expression in osteoblasts. It was further explained that TSA suppressed RANKL-induced osteoclast formation from primary bone marrow-derived macrophages and only effective when present during the early stage of osteoclast differentiation. ${ }^{42}$

\section{Methotrexate}

Methotrexate is a folic acid inhibitor that binds to dihydrofolate reductase thus inhibiting the synthesis of deoxyribonucleic acid (DNA) and ribonucleic acid (RNA). ${ }^{43}$ Methotrexate is currently one of the most common therapies for rheumatoid arthritis patients. Rheumatoid arthritis is often characterized by synovial membrane inflammation and bone destruction, which can be measured by the increment of RANKL/OPG ratio. A study showed that Methotrexate decreased RANKL/OPG ratio and blocked M-CSF-RANKL induced osteoclastogenesis which makes Methotrexate a direct osteoclastogenesis inhibitor. Methotrexate is effective and safe, however, mucositis and oral ulceration can occur as side effects of the treatment, depending on the dosage. It has also been reported that folate deficiency plays a role in predisposing mucositis induced by methotrexate. Due to this, supplementation with folinic acid has been proven effective to help fight the side effects of methotrexate without compromising its therapeutic action. ${ }^{44}$

\section{Denosumab}

Denosumab is a human monoclonal antibody that inhibits bone resorption by mimicking the effects of OPG thus inhibiting the binding of RANKL and RANK, a major signaling in osteoclastogenesis. Denosumab works by targeting RANKL specifically, making it unlikely to affect other regulatory systems. ${ }^{7}$ A study was conducted to evaluate Denosumab's efficacy and safety, it was reported that Denosumab effectively increases the bone mass density (BMD) in postmenopausal women that had been treated with Denosumab for 2 years. ${ }^{45}$ While Denosumab has been shown to be a safe therapy choice, there is a possible side effect that might need further correlation study called frozen bone process. Complete inhibition of bone remodeling process leads to increment of microfractures thus increasing the risk of atypical femoral fracture called the frozen bone process. $^{7}$

\section{Natural Resources as Bone Resorption Inhibitor}

Evidence in studies proved that natural sources including herbal products could participate in these processes thus giving beneficial factors to bone's well-being. A group of vegetables consists of onion, garlic and parsley was reported to have the ability to inhibit bone resorption in ovariectomized rats. Essential oils are taken from thyme, sage, rosemary and other herbs inhibit osteoclast activity thus increasing bone mineral density. Soya bean, usually known for its rich source of isoflavones has shown promising results to use as a therapeutic agent in maintaining bone

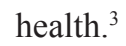

On a more specific level, caffeic acid (3,4-dihydroxycinnamic acids) is a molecule found on a variety of plants, one of which is yam that has an inhibitory effect towards osteoclastogenesis by inhibiting NFKB. ${ }^{4,46}$ It's derivative, caffeic acid phenethyl ester (CAPE) has been shown to have anti-inflammatory component and suppresses osteoclastogenesis. CAPE significantly inhibited osteoclast formation induced by RANKL and proposed as a therapeutic agent for bone related diseases. ${ }^{47}$ 


\section{Onion}

Onion (Allium cepa) is a species member of Allium genus and is a widely used food ingredient. Its beneficial properties have been studied, and evidence showed that onion inhibited bone resorption in rats in a dose dependant manner. ${ }^{48}$ Onion powder solution was reported to inhibit osteoclastogenesis by inhibiting RANKL-induced ERK, p38 MAPK, and NFאB activation but did not affect the differentiation and maturation of cultured osteoblasts. Current studies' data suggest that onion enriched diet might be beneficial to bone's health and prevent bone loss related diseases. $^{49}$

\section{Caffeic acid}

Caffeic acid is one of hydroxycinnamic acids found in green plants and food sources such as berries, potatoes, coffee, carrots and sweet potato that has been studied by scientists to evaluate its beneficial properties. ${ }^{4,50}$ Caffeic acid is a natural resource that has many uses as a therapeutic agent, one of which is to inhibit N-Nitrosation in vitro, which can prevent the formation of mutagenic and carcinogenic compounds. A study in 2011 showed caffeic acid's significant inhibitory ability towards osteoclastogenesis by suppressing NFKB activity. A detailed mechanism of how caffeic acid inhibits osteoclastogenesis remains unclear. ${ }^{4}$ However, a further study explained that caffeic acid did not significantly affect the expression of TRAF 6 while significantly suppressed NFкB's activity in TRAF 6-transfected RANKL-TNF- $\alpha$-induced RAW-D cells. ${ }^{51}$ Apoptotic induction by caffeic acid has also been reported in cancer cells such as SCM1 Gastric, HeLa cervical cancer cells, and lastly, MG-63 osteosarcoma cell line, through a series of caspase activation. ${ }^{52}$

\section{CAPE}

CAPE is an active compound found in the extract of propolis collected by bees from several plant species. CAPE fights viruses, works as an anti-inflammatory, anti-mitogenic, anti-carcinogenic, and an effective immunomodulator. ${ }^{53}$ It has been shown that CAPE has a positive impact on wound healing and inhibits osteoclastic activity, which benefits new bone formation. CAPE does not have a significant influence on the phosphorylation of p38 MAPK, JNK and ERK in the bone marrow cells of mice and fights bone resorption by suppressing RANKL-induced NFאB/NFATc activity and inducing osteoclast apoptosis..$^{54,55}$

\section{Soy isoflavones}

Flavanoids have been reported to enhance bone formation and inhibit bone resorption through various signaling pathways that influence the bone metabolism. Flavanoids can be found in fruits, vegetables, herbs, spices, essential oils, and beverages. Soy isoflavones is a member of the flavanoid family that has been studied for their role in bone health and can be found in soya beans. ${ }^{56}$ Soy isoflavones called as daidzain and genistein often found in soybeans have a similar structure to estrogen and have the ability to bind to the estrogen receptor. A study showed that isoflavones might help postmenopausal women to prevent osteoporosis. ${ }^{57,58}$ In addition, soy isoflavones suppress osteoclastic activities by working like estrogens, inhibiting c-Fos-induced NFATc1 expression. $^{59}$

\section{Other natural resources}

Traditional herbal medicine has been widely used amongst the Chinese and Ayurvedic medical practice. ${ }^{3}$ Turmeric root (Curcuma longa) for example, a staple ingredient in Chinese and Ayurvedic medical practice has an active ingredient called curcumin that has been proven to prevent osteoclastogenesis while also has anti-inflammatory and antioxidant characteristics. ${ }^{60}$ Chinese traditional herbs such as Herba epimedii (Yín Yáng Huò) and Fructus psoraleae (Bǔ Gŭ Zhī) contain components that give similar effects to estrogen that may have the potential to be developed as new phytoestrogens. ${ }^{61}$ Other Chinese traditional herbs called Malva verticillata showed promising results suppressing osteoclast activities by inhibiting c-Fos, NFATc1, the phosphorylation of c-Jun N-terminal kinase, p38, inhibitor of $\kappa \mathrm{B} \alpha(\mathrm{I} \kappa \mathrm{B}) \alpha$ and phospholipase $\gamma 2 .^{62}$ Stewartia koreana, a natural plant product found in Korea also reported having a strong inhibitory effect on osteoclast formation by suppressing the induction of c-Fos and NFATc1 by RANKL. ${ }^{5}$

\section{Conclusion}

Bone resorption process is a complex mechanism that involves various bone metabolism pathways creating mature osteoclast cells. Understanding the mechanisms of bone resorption is one of the keys to treat bone-related diseases. Various treatments have been discovered from synthetic drugs to natural resources. While synthetic drugs are more commonly known for its benefits, it comes with a package of potential side effects. On the other hand, natural 
resources might have the potential to treat bone-related diseases without the harming side effects of synthetic drugs, targeting specific aspects of the osteoclastogenesis pathways to provide suiting therapy for bone related diseases.

\section{References}

1. Patil CS, Kirkwood KL. MAPK P38 MAPK Signaling in OralRelated Diseases. J Dent Res. 2007; 86(9): 812-25.

2. Thouverey C, Caverzasio J. Focus on The P38 MAPK Signaling Pathway in Bone Development and Maintenance. Bonekey Rep. 2015; 4: 711. doi: 10.1038/Bonekey.2015.80.

3. Putnam SE, Scutt AM, Bicknell K, Priestley CM, Williamson EM. Natural Products as Alternative Treatments for Metabolic Bone Disorders and for Maintenance of Bone Health. Phytother Res. 2007; 21(2): 99-112.

4. Sandra F, Kukita T, Tang QY, Iijima T. Cafeic Acid Inhibits Nfkappab Activation of Osteoclastogenesis Signaling Pathway. Indones Biomed J. 2011; 3(3): 216-22.

5. Park CK, Kim HJ, Kwak HB, Lee TH, Bang MH, Kim CM, et al. Inhibitory Effects of Stewartia koreana on Osteoclast Differentiation and Bone Resorption. Int Immunopharmacol. 2007; 7(12): 1507-16.

6. Fadanelli ME, Bone HG. Combining Bisphosphonates with Hormone Therapy for Postmenopausal Osteoporosis. Treat Endocrinol. 2004; 3(6): 361-9.

7. Miyazaki T, Tokimura F, Tanaka S. A Review of Denosumab for The Treatment of Osteoporosis. Patient Prefer Adherence. 2014; 8: 46371.

8. Revu S, Neregård P, Klint E, Korotkova M, Catrina AI. Synovial Membrane Immunohistology in Early-Untreated Rheumatoid Arthritis Reveals High Expression of Catabolic Bone Markers That is Modulated by Methotrexate. Arthritis Res Ther. 2013; 15(6): $1-10$.

9. Kwak HB, Kim JY, Kim KJ, Choi MK, Kim JJ, Kim KM, et al. Risedronate Directly Inhibits Osteoclast Differentiation and Inflammatory Bone Loss. Biol Pharm Bull. 2009; 32(7): 1193-8.

10. Barbato L, Francioni E, Bianchi M, Mascitelli E, Brancato L, Duvina $\mathrm{M}$, et al. Periodontitis and Bone Metabolism. Clin Cases Miner Bone Metab. 2015; 12(2): 174-7.

11. Gruber R. Cell Biology of Osteoimmunology. Wien Med Wochenschr. 2010; 160(17-18): 438-45.

12. Sandra F, Hendarmin L, Nakamura S. Osteoprotegerin (OPG) Binds with Tumor Necrosis Factor-Related Apoptosis-Inducing Ligand (TRAIL): Suppression of TRAIL-Induced Apoptosis in Ameloblastomas. Oral Oncol. 2006; 42(2): 415-20.

13. Kukita A, Ichigi Y, Takigawa I, Watanabe T, Kukita T, Miyamoto H. Infection of RANKL-Primed RAW-D Macrophages with Porphyromonas Gingivalis Promotes Osteoclastogenesis in A TNF$\alpha$-Independent Manner. Plos One. 2012; 7(6): 1-12. doi:10.1371/ Journal.Pone.0038500. E38500.

14. Kim JH, Kim N. Signaling Pathways in Osteoclast Differentiation. Chonnam Med J. 2016; 52(1): 12-7.

15. Soysa NS, Alles N, Aoki K, Ohya K. Osteoclast Formation and Differentiation: An Overview. J Med Dent Sci. 2012; 59(3): 65-74.

16. Chambers TJ. Regulation of The Differentiation and Function of Osteoclasts. J Pathol. 2000; 192(1): 4-13.

17. Boyce BF, Xing L. Functions Of RANKL/RANK/OPG in Bone Modeling and Remodeling. Arch Biochem Biophys. 2008; 473(2):
$139-46$.

18. Bell NH. RANK Ligand and The Regulation of Skeletal Remodeling. J Clin Invest. 2003; 111(8): 1120-2.

19. Boyle WJ, Simonet WS, Lacey DL. Osteoclast Differentiation and Activation. Nature. 2003; 423(6937): 337-42.

20. Kim N, Kadono Y, Takami M, Lee J, Lee SH, Okada F, et al. Osteoclast Differentiation Independent of The TRANCE-RANK-TRAF6 Axis. J Exp Med. 2005; 202(5): 589-95.

21. Kobayashi N, Kadono Y, Naito A, Matsumoto K, Yamamoto T, Tanaka $\mathrm{S}$, et al. Segregation of TRAF6-Mediated Signaling Pathways Clarifies Its Role in Osteoclastogenesis. EMBO J. 2001; 20(6): 1271-80.

22. Kawamura N, Kugimiya F, Oshima Y, Ohba S, Ikeda T, Saito T, et al. Akt1 in Osteoblasts and Osteoclasts Controls Bone Remodeling. Plos One. 2007; 2(10): 1-11.

23. Maruotti N, Grano M, Colucci S. Osteoclastogenesis and Arthritis. Clin Exp Med. 2011; 11(3): 137-45.

24. Wada T, Nakashima T, Hiroshi N, Penninger JM. RANKL-RANK Signaling in Osteoclastogenesis and Bone Disease. Trends Mol Med. 2006; 12(1): 17-25.

25. Aliprantis AO, Ueki Y, Sulyanto R, Park A, Sigrist KS, Sharma $\mathrm{SM}$, et al. Nfatc1 in Mice Represses Osteoprotegerin During Osteoclastogenesis and Dissociates Systemic Osteopenia From Inflammation in Cherubism. J Clin Invest. 2008; 118: 3775-89.

26. Walsh MC, Choi Y. Biology Of The RANKL-RANK-OPG System In Immunity, Bone, And Beyond. Front Immunol. 2014; 5: 1-11. doi: 10.3389/fimmu.2014.00511.

27. Ding X, Boney-Montoya J, Owen BM, Bookout AL, Coate C, Mangelsdorf DJ, et al. Bklotho is Required for Fibroblast Growth Factor 21 Effects on Growth and Metabolism. Cell Metab. 2012; 16(3): 387-93.

28. Weitzmann MN, Pacifici R. The Role of T Lymphocytes in Bone Metabolism. Immunol Rev. 2005; 208: 154-68.

29. Wu L, Guo Q, Yang J, Ni B. Tumor Necrosis Factor Alpha Promotes Osteoclast Formation via PI3K/Akt Pathway-Mediated Blimp1 Expression Upregulation. J Cell Biochem. 2016; 118(6): 1308-15.

30. Koga T, Kodama T, Ochi S, Shinohara M, Sato K, Takai T, et al. Pathological Role of Osteoclast Costimulation in Arthritis-Induced Bone Loss. PNAS. 2007; 104(27): 11394-9.

31. Faccio R, Takeshita S, Zallone A, Ross F, Teitelbaum S. C-Fms and The Avß3 Integrin Collaborate During Osteoclast Differentiation. J Clin Invest. 2003; 111(5): 749-58.

32. Catrina AI, Trollmo C, Klint E, Engstrom M, Lampa J, Hermansson Y, et al. Evidence That Anti-tumor Necrosis Factor Therapy with Both Etanercept and Infliximab Induces Apoptosis in Macrophages, but Not Lymphocytes, in Rheumatoid Arthritis Joints Extended Report. Arthritis Rheum. 2005; 52(1): 61-72.

33. Pers J, Saraux A, Pierre R, Youinou P. Anti-TNF- $\alpha$ Immunotherapy is Inflammation without Clinical Attachment Loss in Subjects with Rheumatoid Arthritis. J Periodontol. 2008; 79(9): 1645-51.

34. Yamashita T, Takahashi N, Udagawa N. New Roles of Osteoblasts Involved in Osteoclast Differentiation. 2012; 3(11): 175-81.

35. Ross F. M-CSF, C-Fms, and Signaling in Osteoclasts and Their Precursors. Ann NY Acad Sci. 2006; 1068(1): 110-6.

36. Newton A. Protein Kinase C: Structural and Spatial Regulation by Phosphorylation, Cofactors, and Macromolecular Interactions. Chem Rev. 2001; 101(8): 2353-64.

37. Russell RGG. Pharmacological Diversity A-mong Drugs That Inhibit Bone Resorption. Curr Opin Pharmacol. 2015; 22: 115-30. 
38. Drake MT, Clarke BL, Khosla S. Bisphosphonates: Mechanism of Action and Role in Clinical Practice. Mayo Clin Proc. 2008; 83(9): 1032-45.

39. Kim YH, Kim G, Baek JH. Inhibitory Action of Bisphosphonates on Bone Resorption Does Not Involve The Regulation Of RANKL and OPG Expression. Exp Mol Med. 2002; 34(2): 145-51.

40. Drake MT, Clarke BL, Khosla S. Bisphosphonates: Mechanism of Action and Role in Clinical Practice. Mayo Clin Proc. 2009; 83(9): 1032-45

41. Watts NB, Diab DL. Long-Term Use of Bisphosphonates in Osteoporosis. J Clin Endocrinol Metab. 2010; 95(4): 1555-65.

42. Kim H, Ha H, Lee J, Jung K, Yang D, Woo KM, et al. Trichostatin A Inhibits Osteoclastogenesis and Bone Resorption by Suppressing The Induction of C-Fos By RANKL. Eur J Pharmacol. 2009; 623(13): 22-9.

43. Yi T, Baek J. Trichostatin A-Mediated Upregulation of P21 Osteoclast Apoptosis. Exp Mol Med. 2007; 39(2): 213-21.

44. Vigushin DM, Ali S, Pace PE, Mirsaidi N, Ito K, Adcock I, et al. Trichostatin A is A Histone Deacetylase Inhibitor with Potent Antitumor Activity Against Breast Cancer in vivo. Clin Cancer Res. 2001; 7(4): 971-6.

45. Lopez-Olivo MA, Siddhanamatha HR, Shea B, Tugwell P, Wells GA, Suarez-Almazor ME. Methotrexate for Treating Rheumatoid Arthritis. Cochrane Database Syst Rev. 2014; (6): CD000957. doi: 10.1002/14651858.CD000957.pub2.

46. Deeming GMJ, Collingwood J, Pemberton MN. Methotrexate and Oral Ulceration. Br Dent J. 2005; 198(2): 83-5.

47. Lewiecki EM, Miller PD, Mcclung MR, Cohen SB, Bolognese MA, Liu Y, et al. Two-Year Treatment with Denosumab (AMG 162) in A Randomized Phase 2 Study of Postmenopausal Women with Low BMD. Journals Bone Miner Res. 2007; 22(12): 1832-41.

48. Quan YT, Kukita T, Ushijima Y, Kukita A, Nagata K, Sandra F, et al. Regulation of Osteoclastogenesis by Simon Extracts Composed of Caffeic Acid and Related Compounds: Successful Suppression of Bone Destruction Accompanied with Adjuvant-Induced Arthritis in Rats. Histochem Cell Biol. 2006; 125(3): 215-25.

49. Ha J, Choi H, Lee Y, Lee ZH, Kim H. International Immunopharmacology Caffeic Acid Phenethyl Ester Inhibits Osteoclastogenesis by Suppressing Nfkb and Downregulating Nfatc1 And C-Fos. Int Immunopharmacol; 2009; 9(6): 774-80.

50. Mirabeau TY, Samson ES. Effect of Allium Cepa and Allium Sativum on Some Immunological Cells in Rats. Afr J Tradit Complement Altern Med. 2012; 9(3): 374-9.

51. Tang C, Huang T, Chang C, Fu W. Water Solution of Onion Crude Powder Inhibits RANKL-Induced Osteoclastogenesis Through ERK, P38 and Nfkb Pathways. Osteoporos Int. 2009; 20(1): 93-103.
52. Silva T, Oliveira C, Borges F. Caffeic Acid Derivatives, Analogs and Applications: A Patent Review. Expert Opin Ther Patents. 2014; 24(11): 1257-70.

53. Sandra F, Kukita T, Muta T, Iijima T. Caffeic Acid Inhibited Receptor Activator of Nuclear Factor $\mathrm{Kb}$ Ligand (RANKL)-Tumor Necrosis Factor (TNF) A-TNF Receptor Associated Factor (TRAF) 6 Induced Osteoclastogenesis Pathway. Indones Biomed J. 2013; 5(3): 173-8.

54. Sandra F, Sidharta MA. Caffeic Acid Induced Apoptosis in MG63 Osteosarcoma Cells Through Activation of Caspases. Mol Cell Biomed Sci. 2017; 1(1): 28-33.

55. Kuo Y, Jim W, Su L, Chung C, Lin C. Caffeic Acid Phenethyl Ester is A Potential Therapeutic Agent For Oral Cancer. Int J Mol Sci. 2015; 16(5): 10748-66.

56. Ang ESM, Pavlos NJ, Chai LY, Qi M, Cheng TS, Steer JH, et al. Caffeic Acid Phenethyl Ester, An Active Component of Honeybee Propolis Attenuates Osteoclastogenesis and Bone Resorption via The Suppression of RANKL-Induced Nfkb and NFAT Activity. J Cell Physiol. 2009; 221(3): 642-9.

57. Hung MW, Shiao MS, Tsai LC, Chang GG, Chang TC. Apoptotic Effect of Caffeic Acid Phenethyl Ester and Its Ester and Amide Analogues in Human Cervical Cancer ME180 Cells. Anticancer Res. 2003; 23(6C): 4773-80.

58. Weaver CM, Alekel L, Ward WE, Ronis MJ. Flavonoid Intake and Bone Health Connie. J Nutr Gerontol Geriatr. 2012; 31(3): 239-53.

59. Taku K, Melby MK, Nishi N, Omori T, Kurzer MS. Soy Isoflavones for Osteoporosis: An Evidence-Based Approach. Maturitas. 2011; 70(4): 333-8.

60. Kostelac D, Rechkemmer G, Briviba K. Phytoestrogens Modulate Binding Response of Estrogen Receptors Alpha and Beta to The Estrogen Response Element. J Agric Food Chem. 2003; 51(26): 7632-5.

61. Karieb S, Fox SW. Phytoestrogens Directly Inhibit TNF-A-Induced Bone Resorption in RAW264.7 Cells by Suppressing C-FosInduced Nfatc1 Expression. J Cell Biochem. 2011; 112(2): 47687.

62. Bharti AC, Takada Y, Aggarwal BB. Curcumin (Diferuloylmethane) Inhibits Receptor Activator of NF-Kb Ligand-Induced NF$\mathrm{Kb}$ Activation in Osteoclast Precursors and Suppresses Osteoclastogenesis. J Immunol. 2004; 172(10): 5940-7.

63. Leung P, Siu W. Herbal Treatment for Osteoporosis: A Current Review. J Tradit Complement Med. 2013; 3(2): 82-7.

64. Shim K, Lee C, Yim N, Ha H, Ma JY. A Water Extract of Malva Verticillata Seeds Suppresses Osteoclastogenesis and Bone Resorption Stimulated by RANK Ligand. BMC Complement Altern Med. 2016; 16(1): 332. doi: 10.1186/S12906-016-1295-6. 